\title{
Global Trend in Visual Impairment and Blindness, Changing Pattern, Challenges and Facts
}

\section{Pradeep Bastola*}

Associate Professor, Head of Department in Ophthalmology, Nepalgunj Medical College and Teaching Hospital, Kohalpur, Nepal

*Corresponding Author: Pradeep Bastola, Associate Professor, Head of Department in Ophthalmology, Nepalgunj Medical College and Teaching Hospital, Kohalpur, Nepal.

Received: October 23, 2019; Published: November 01, 2019

DOI: $10.31080 /$ ASOP.2020.03.0079

In a world built on the ability to see, vision, the most dominant of our all senses $(80 \%)$ is critical at every moment of our lives. According to the World Health Organization (WHO), World Report on Vision (October 2019), globally 2.2 billion people have some kind of visual impairment or blindness and 1 billion of them could have been addressed or prevented. Everyone if they live long will experience some kind of ocular problem in their lifetime and will need an appropriate treatment option for the same. Visual impairment occurs when an eye condition affects the visual system and one or more of its vision functions. A person who wears glasses or contact lenses to compensate for their visual impairment still possess visual impairment.

The pattern of visual impairment has changed over the last 30 years considerably due to concrete policies, strategies and guidelines made by WHO, IAPB, Ministries of Health to combat visual impairment and blindness related disability. Important developments were VISION 2020, Global Elimination of Trachoma (GET), Combating Onchocerciasis (Mass drug distribution), SAFE strategies to eliminate blinding Trachoma, evolution in the eye care services, WHO adopted new policies, National Vitamin A Distribution Programs, Ministries of Health involvement to address visual impairment and blindness, Non-governmental Organizations (NGOs) support in eye care, longer life expectancy, sedentary life style, obesity, smoking and non-communicable diseases have had real impact in decreasing global burden of visual impairment and blindness and at the same time also changed the pattern of visual impairment and blindness across the globe.

The world health organization in the year 2004 estimated vision loss caused $3.9 \%$ of total global burden of disease when measured in disability adjusted life in years. The main causes of visual impairment and blindness were then Cataract, Trachoma, Glaucoma, Retinal Problems, Age related macular degeneration, Infectious eye diseases, Vitamin A deficiency, River blindness and Refractive Error. In the year when Mid Term VISION 2020 survey was done. The estimates 285 million people have some kind of visual impairment and 39 million people were blind. The commonest causes for visual impairment (2010) were refractive error followed by cataract, while the commonest cause for blindness still remained cataract. $65 \%$ of the adults above 50 years of age had some kind of visual impairment and $82 \%$ blinds in the same age group.

Now, with VISION 2020 almost coming to its end and Global Action Plan (2014 - 2019 ending soon), the estimated number for visual impairment and blindness are 253 million globally and 36 million of them are blind. 124 million people have uncorrected refractive error while 65 million have cataracts, interestingly 1.29 billion people need near glasses to read. The challenge which lies ahead of us at present with the Global Report on Vision is the alarming rise in non-communicable diseases like Diabetes and Hypertension. The commoner cause of visual impairment globally now are refractive error, cataract, glaucoma, diabetic retinopathy and age related macular degeneration. The projected number of diabetic population for the year 2030 and 2050 is much more than the current 424 million (approximately), and it is a known fact that a diabetic in spite of excellent blood sugar control is liable to develop retinal changes over a period of time.

With the given numbers we should also be aware that $90 \%$ of these ocular morbidities are in low to middle income countries and $75-80 \%$ of them are avoidable, treatable or curable. Just for an example, it is estimated that approximately $15.3 \%$ of the blind population reside in the Africa region and 26.3 million people have 
visual impairment, this region has $73 \%$ proportionately blind people than any other region and has the highest out of pocket expenditure for eye care facilities globally. The challenges we have in providing eye care services in these poorer regions are;

- $\quad$ Primary Eye Care coverage still incomplete and dependent on NGOs.

- $\quad$ Eye care and health taken as only a form of morbidity.

- $\quad$ Lack of human resources/infrastructures.

- $\quad$ Lack of strong leadership by the governments with regards to Primary Eye Care.

- Vertical approach not succeeding, need of an integration with the existing primary health care in line with Universal Health Coverage.

- Various Sustainable Development Goals (SDGs) eight of them, align directly with eye care, need of advocacy regarding it.

The good news for us during the last 3 decades with considerable work in neglected tropical eye diseases like trachoma and onchocerciasis. Trachoma no longer is a public health blinding eye disease in majority of the countries, countries like Nepal, Cambodia, Ghana have eliminated trachoma. Onchocerciasis also due to strong advocacy and mass distribution of Ivermectin tablets is no longer a blinding disease in Africa region.

October is a month when we have World Sight Day falling on second Thursday each year, and also is the month where we should unite our efforts to work in the field of Ophthalmology for advocacy and awareness and also to provide promotive, preventive, curative, palliative and rehabilitative eye care. The theme for this year's world sight day is "Vision First" with the given backdrop lets work together to leave no one left behind visually impaired or blind and prioritize Vision as the most common sense used by us the humans.

Volume 3 Issue 1 January 2020

(C) All rights are reserved by Pradeep Bastola. 